\title{
A Rare Case of Giant Solid Hemangioblastoma Accompanied with Hemophilia Type A
}

\author{
Masaki Ujihara, ${ }_{1}$ Yoshinori Maki, ${ }^{1}$ Masaki Chin, ${ }^{1}$ Kensuke Takada, ${ }^{1}$ Yoshitaka Kurosaki, ${ }^{1}$ and Sen Yamagata ${ }^{1}$
}

\begin{abstract}
The surgical removal of giant solid hemangioblastoma involves a high risk of perioperative bleeding and requires attentive hemostasis. Here, we present a case of a giant solid hemangioblastoma accompanied with hemophilia which was previously undiagnosed. A 35-year-old man without any past medical history was admitted with diplopia and ocular motility disorder. computed tomography (CT) and magnetic resonance imaging $(\mathrm{MRI})$ revealed obstructive hydrocephalus and a solid giant tumor of more than $4.0 \mathrm{~cm}$ in diameter in the right cerebellopontine angle (CPA). Hemangioblastoma was suspected on cerebral angiography. After ventriculoperitoneal shunt for obstructive hydrocephalus, oozing from the skin incision continued for several days. Hemophilia type A was diagnosed based on the result of laboratory blood coagulability examination. Supplemental administration of factor VIII and coil embolization of the feeding arteries of the lesion on the CPA were performed, and the tumor was subtotally resected without hemorrhagic complications. The histopathological diagnosis was hemangioblastoma. We report this case to emphasize the importance not to overlook previously undiagnosed coagulopathy before surgical excision of hemangioblastoma. And, with appropriate perioperative management for coagulopathy, surgical treatment involving a high risk of perioperative bleeding can be safely undertaken.
\end{abstract}

Keywords: hemangioblastoma, hemophilia type A, perioperative treatment, factor VIII

\section{Introduction}

Hemangioblastoma is a vascularized benign tumor of the central nervous system, and commonly occurs in the cerebellum, brainstem, or spinal cord. ${ }^{1)}$ Hemangioblastoma is varied in appearance, including solid, solid-cystic, or mainly cystic with a mural nidus. ${ }^{2)}$ The treatment of solid hemangioblastoma is complex and of a high mortality and morbidity due to the risk of perioperative bleeding. ${ }^{3)}$ It has also been postulated that the bleeding risk of solid hemangioblastoma is mainly related to the size of hemangioblastoma. ${ }^{4)}$ The size

${ }^{1}$ Department of Neurosurgery, Kurashiki Central Hospital, Kurashiki, Okayama, Japan

Received: March 8, 2018; Accepted: May 30, 2018

Online September 13, 2018

Copyright $(2018$ by The Japan Neurosurgical Society This work is licensed under a Creative Commons AttributionNonCommercial-NoDerivatives International License. of the hemangioblastoma is classified into three groups: small $(<3 \mathrm{~cm})$, large $(3.1-4 \mathrm{~cm})$, and giant $(>4 \mathrm{~cm}){ }^{2,4,5)}$ The excision of a giant solid hemangioblastoma is particularly challenging, ${ }^{2)}$ especially in cases accompanied with coagulation disorders. We herein present a case of a giant solid hemangioblastoma accompanied with hemophilia type A.

\section{Case Report}

A 35-year-old man was admitted with diplopia and ocular motility disorder. The patient had experienced episodes of dizziness and nausea for 2 weeks prior to presentation. The patient had no personal or family history of bleeding diathesis, and laboratory blood coagulability examination was within normal range: activated partial thromboplastin time (APTT) was $43.5 \mathrm{~s}$ (normal range, 30.3-45.4 s) and prothrombin time was $12.7 \mathrm{~s}$ (normal range, 11.6-14.0 s). CT revealed a mass in the right cerebellopontine angle (CPA) and hydrocephalus. The mass in the right CPA was $4.2 \times 4.1 \times 3.5$ $\mathrm{cm}^{3}$ and was compressing the fourth ventricle. The lesion was demonstrated on T1-weighted gadolinium-enhanced magnetic resonance imaging (MRI) (Fig. 1). Emergent ventricular drainage was performed on the day of admission, and there was no apparent evidence of a hemorrhagic event.

Three hours postoperatively, the drainage fluid was observed as bloody. We observed slight intraventricular hemorrhage on computed tomography (CT), but no surgical treatment was needed. Cerebral angiography confirmed a highly vascular lesion with feeding vessels from the right posterior inferior cerebellar artery (PICA) and the basilar artery (BA), and we suspected cerebellar hemangioblastoma (Fig. 2A). A week after the emergent ventricular drainage, we performed placement of a ventriculoperitoneal shunt to resolve hydrocephalus. After the shunt operation, oozing from the skin incision of the head and abdomen persisted for several days. The coagulability was examined again. APTT was 48.3 s (normal range, 30.3$45.4 \mathrm{~s}$ ) and coagulation factor VIII activity was $33.0 \%$ (normal range: $80.0-140.0 \%$ ), thus hemophilia type A was diagnosed.

Because the lesion of the right CPA compressed the brainstem and fourth ventricle, we considered surgical treatment. From the day before the surgery, supplemental administration of coagulation factor VIII was initiated to maintain coagulation factor activity $>80 \%$, and we performed coil embolization of the main feeders from the right PICA and the BA to reduce the blood flow of the lesion in the right CPA. Contrast enhancement was observed in the feeders from the right superior cerebellar artery (SCA), which was not apparent prior to embolization. As the feeders from the 


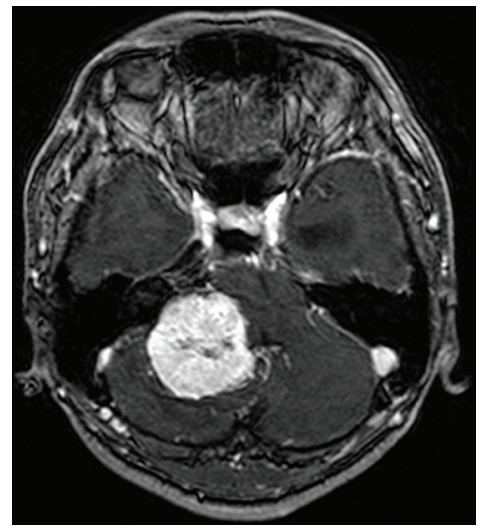

Fig. 1 T1-weighted gadolinium-enhanced MRI showing the mass lesion in the right cerebellopontine angle. The fourth ventricle was compressed by the mass lesion.
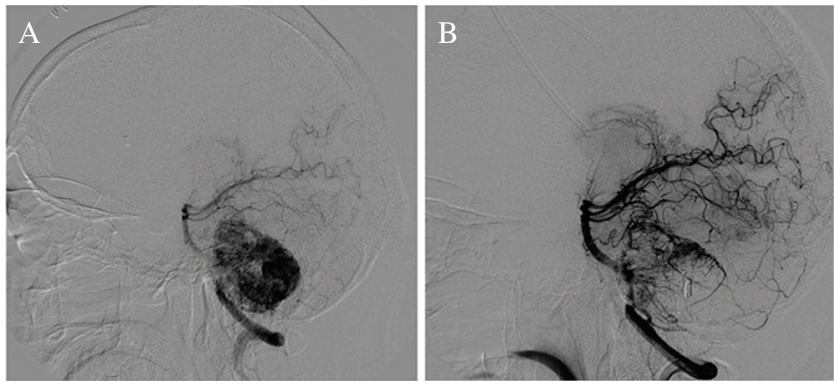

Fig. 2 (A) Cerebral angiography demonstrating feeding vessels from the right posterior inferior cerebellar artery (PICA) and basilar artery (BA). Prominent contrast enhancement was observed. (B) After embolization of the feeders from the right PICA and BA, tumor was still fed by the right superior cerebellar artery (SCA).

right SCA were thin, embolization was not performed in the right SCA (Fig. 2B). Following the endovascular treatment, we performed surgical resection of the right CPA with a right lateral suboccipital approach. The tumor adhered tightly to the cranial nerves, and thus we resected the tumor in a piecemeal fashion. Intraoperative bleeding, possibly from the residual feeders located behind the tumor, was confirmed but controlled. The tumor was subtotally removed, and a postoperative hemorrhagic event did not occur.

Supplemental administration of coagulation factor VIII was ceased on postoperative day 6 . The histopathological diagnosis was hemangioblastoma, and dysacusis was residual following the surgery. The patient was discharged from the hospital after 10 weeks of rehabilitation. A bleeding event requiring hospitalization did not occur following discharge.

\section{Discussion}

We report a case of hemangioblastoma accompanied with hemophilia type A. In our case, hemophilia type A was diagnosed with clinical signs of bleeding tendency and repeated evaluation of coagulability, and the supplemental administration of coagulation factor was needed for perioperative management.
Hemophilia type A, caused by a deficiency of coagulation factor VIII, is categorized based on the coagulation factor activity as severe ( $<1 \%$ of normal), moderate ( $1-5 \%$ of normal) or mild $\left(>5 \%-<40 \%\right.$ of normal), ${ }^{6}$ from which the latter diagnosis comprises of approximately $30-45 \%$ of patients. The diagnosis in our case was mild hemophilia with a coagulation factor activity of $33.0 \%$. Similar to our case, patients with mild hemophilia often remain undiagnosed when there is an absence of a history of trauma or surgery. ${ }^{7)}$ Moreover, approximately one-third of hemophiliacs do not have family history of hemophilia. ${ }^{8)}$ Therefore, mild hemophilia cannot be excluded solely based on a lack of family history or the patient's age, and surgical treatment without the supplemental administration of the deficient coagulation factor can be fatal. ${ }^{9}$

In the guideline of World Federation of Hemophilia, surgical treatment for hemophiliacs is recommended in the hemophilia treatment center with laboratory support of monitoring clotting factor level and inhibitor examination. Administration of coagulation factors is also needed for hemophiliacs. Bolus injection followed by continuous infusion can produce steadystate levels of factor VIII. In the absence of an inhibitor, initial bolus dose is calculated according to following formula: bolus dose $(\mathrm{IU})=0.5 \times$ desired factor VIII rise (percent of normal) $\times$ body weight $(\mathrm{kg}){ }^{8}{ }^{8}$ Dose of approximately $4 \mathrm{IU} / \mathrm{kg} / \mathrm{h}$ of factor VIII will often maintain the level initially achieved by the bolus transfusion, which should be adjusted based on frequent monitoring of factor VIII. ${ }^{10)}$ For major surgery, factor VIII activity should be maintained $80-100 \%$ preoperatively, $40-60 \%$ postoperatively for $4-6$ days, and $30-50 \%$ postoperatively for 7-14 days. ${ }^{8}$ Because factor VIII activity was above $30 \%$ before supplemental administration in our case, we kept the administration of factor VIII until postoperatively at day 6 , and there was no hemorrhagic complication.

In our patient, the result of APTT in blood examination was initially within normal range probably because hemophilia type A was not severe. However, it seems important to examine again coagulability in case hemorrhagic diathesis is suspected.

It is known that hemangioblastoma produces excessive erythropoietin and can cause paraneoplastic erythrocytosis, ${ }^{11,12)}$ but little has been reported about hemangioblastoma accompanied by hemophilia. A rare case of a facial arteriovenous malformation associated with consumption coagulopathy has been reported. ${ }^{13)}$ Although in our case, coagulopathy might have occurred due to a hypervascular lesion, a deficiency of coagulation factor VIII remained after tumor resection. Therefore, it seemed likely that hemophilia coexisted incidentally with hemangioblastoma in our patient.

The careful attention of hemostasis is essential for surgical treatment of hemangioblastoma due to hypervascularity. With the progress of preoperative radiological evaluation and microsurgical techniques, and with the assistance of endovascular embolization, the postoperative mortality and morbidity have improved. ${ }^{2-4,14,15)}$ However, unsuccessful hemostasis is still a factor for high mortality and morbidity. ${ }^{15)}$ The surgical resection of hemangioblastoma increases in difficulty with an increasing tumor size, with poorer surgical outcomes especially in cases of giant hemangioblastoma of more than $4.0 \mathrm{~cm} .{ }^{2}$ Furthermore, the hemangioblastoma in our case was 
accompanied with hemophilia, which further adds difficulty in achieving hemostasis.

En-bloc resection is preferred to minimalize intraoperative bleeding in contrast to the piecemeal resection, which can result in major bleeding. ${ }^{15)}$ However, en-bloc resection becomes difficult as the size of a hemangioblastoma increases, and in cases where the hemangioblastoma adheres to the cranial nerves, piecemeal resection must be undertaken to avoid damage to the nerves, as seen in our case.

If mild hemophilia type A was not disclosed preoperatively in our case, we could intraoperatively encounter unexpected hemorrhagic diathesis. In such case, following disorders should be considered, such as multiple transfusions of bank blood, intravascular coagulation, incompatible transfusion, undetected anticoagulant therapy, platelet deficiency, shock with anoxia, and unsuspected mild hemophilia. ${ }^{16)}$ To detect the cause of intraoperative unexpected hemorrhagic diathesis, we should immediately confirm medications of the patient, coagulation function, and blood type of transfusion. Adequate transfusion should be initiated if necessary. In case that we cannot control the bleeding, embolization in a hybrid surgical room can be an option.

\section{Conclusions}

We presented the first known case of giant solid hemangioblastoma accompanied by hemophilia type A. In our case, hemophilia type A was diagnosed before the surgical removal of hemangioblastoma with clinical signs of bleeding tendency and repeated evaluation of coagulability. And hemostasis was achieved through the supplemental administration of coagulation factor VIII. If undiagnosed coagulopathy is suspected, blood coagulability should be examined. With appropriate perioperative management for coagulopathy, surgical treatment involving a high risk of perioperative bleeding can be safely undertaken.

\section{Acknowledgment}

We thank Paul Williams (Kurashiki Central Hospital) for his extensive proofreading and advice.

\section{Conflicts of Interest Disclosure}

The authors report no conflict of interest concerning the findings specified in this paper.

\section{Compliance with Ethical Standards and Informed Consent}

The authors obtained informed consent from the patient and his family.

\section{References}

1) Neumann HP, Eggert HR, Weigel K, Friedburg H, Wiestler OD, Schollmeyer P: Hemangioblastomas of the central nervous system. A 10-year study with special reference to von Hippel-Lindau syndrome. J Neurosurg 70: 24-30, 1989

2) Rachinger J, Buslei R, Prell J, Strauss C: Solid haemangioblastomas of the CNS: a review of 17 consecutive cases. Neurosurg Rev 32: 37-47; discussion 47-48, 2009

3) Young S, Richardson AE: Solid haemangioblastomas of the posterior fossa: radiological features and results of surgery. J Neurol Neurosurg Psychiatry 50: 155-158, 1987

4) Wan JQ, Cui H, Wang Y: Surgical management of large solid hemangioblastomas of the posterior fossa. J Clin Neurosci 18: 39-42, 2011

5) Zhou LF, Du G, Mao Y, Zhang R: Diagnosis and surgical treatment of brainstem hemangioblastomas. Surg Neurol 63: 307-315; discussion 315-316, 2005

6) White GC, Rosendaal F, Aledort LM, Lusher JM, Rothschild C, Ingerslev J; Factor VIII and Factor IX Subcommittee: Definitions in hemophilia. Recommendation of the scientific subcommittee on factor VIII and factor IX of the scientific and standardization committee of the International Society on Thrombosis and Haemostasis. Thromb Haemost 85: 560, 2001

7) Venkateswaran L, Wilimas JA, Jones DJ, Nuss R: Mild hemophilia in children: prevalence, complications, and treatment. J Pediatr Hematol Oncol 20: 32-35, 1998

8) Srivastava A, Brewer AK, Mauser-Bunschoten EP, et al.: Guidelines for the management of hemophilia. Haemophilia 19: e1-e47, 2013

9) Kitchens CS: Occult hemophilia. Johns Hopkins Med J 146: 255-259, 1980

10) Holme PA, Tjønnfjord GE, Batorova A: Continuous infusion of coagulation factor concentrates during intensive treatment. Haemophilia 24: 24-32, 2018

11) Zec N, Cera P, Towfighi J: Extramedullary hematopoiesis in cerebellar hemangioblastoma. Neurosurgery 29: 34-37, 1991

12) Tachibana $O$, Yamashima T, Yamashita J: Immunohistochemical study of erythropoietin in cerebellar hemangioblastomas associated with secondary polycythemia. Neurosurgery 28: 24-26, 1991

13) Akimura T, Katoh S, Nomura S, Yamashita T: Facial arteriovenous malformation associated with consumption coagulopathy treated by embolization: report of case. J Oral Maxillofac Surg 52: 1318-1321, 1994

14) Zhou L, Du G: Diagnosis and surgical treatment of posterior fossa solid hemangioblastomas. Chin Med J (Engl) 113: 129-132, 2000

15) Cui H, Zou J, Bao YH, Wang MS, Wang Y: Surgical treatment of solid hemangioblastomas of the posterior fossa: a report of 28 cases. Oncol Lett 13: 1125-1130, 2017

16) Coy J, Bivins BA, Belin RP: Surgical procedures in unsuspected hemophilia. Arch Surg 109: 835-836, 1974

Corresponding author:

Masaki Ujihara, MD, Department of Neurosurgery, Kurashiki Central Hospital, 1-1-1 Miwa, Kurashiki, Okayama, Japan.

$\bowtie$ ujiharam0817@gmail.com 